\title{
Distribution-Free Discriminant Analysis
}

\author{
Tom Burr \\ Justin Doak
}

\section{DISCLAIMER}

This report was prepared as an account of work sponsored by an agency of the United States Government. Neither the United States Government nor any agency thereof, nor any of their employees, makes any warranty, express or implied, or assumes any legal liability or responsibility for the accuracy, completeness, or usefulness of any information, apparatus, product, or process disclosed, or represents that its use would not infringe privately owned rights. Reference herein to any specific commercial product, process, or service by trade name, trademark, manufacturer, or otherwise does not necessarily constitute or imply its endorsement, recommendation, or favoring by the United States Government or any agency thereor. The views and opinions of authors expressed herein do not necessarily state or reflect those of the United States Government or any agency thereof.

\section{Los Alamos




\section{DISCLAMMER}

Portions of this document may be illegible in electronic image products. Images are produced from the best available original document. 


\title{
Distribution-Free Discriminant Analysis
}

\author{
Tom Burr and Justin Doak
}

\section{Abstract}

This report describes our experience in implementing a non-parametric (distribution-free) discriminant analysis module for use in a wide range of pattern recognition problems. Issues discussed include performance results on both real and simulated data sets, comparisons to other methods, and the computational environment. In some cases, this module performs better than other existing methods. Nearly all cases can benefit from the application of multiple methods.

\subsection{Introduction}

We consider the typical pattern recognition problem (also called classification problem), in which the data are assumed to consist of $n$ cases of $(C, \mathbf{X})$ pairs where $C \in(1,2, \ldots, J)$ is the class and $\mathbf{X}$ is a $p$-dimensional feature (predictor) vector. The goal is to use $\mathbf{X}$ to predict the class $C$. There are many traditional and modern approaches to pattern recognition. Many of these, including Fisher's Linear Discriminant, attempt to estimate the probability density of a feature vector, $\mathbf{X}$, given some class [i.e., the class conditional probability, $P(\mathbf{X} \mid C)$ ] by assuming some convenient distribution for $\mathbf{X}$. We focus on methods of estimating densities that make minimal assumptions about the distribution. Such methods are typically called non-parametric or distribution-free methods; thus, we refer to our work as distribution-free discriminant analysis (DFDA). Probability density estimation was invented during the 1950 s to apply non-parametric pattern recognition techniques. However, nearly all work has focused on the case in which all the features are realvalued (continuous features), and even in that case good implementations are rare. (We only know of one implementation, available as a probabilistic neural network, in a commercial product called NeuralWare.) Therefore, another focus of our work is to handle not only real-valued but also categorical (unordered and ordered) features.

The remainder of this paper will be both an introduction to distribution-free density estimation techniques and details of our specific implementation, including software use and installation. 


\subsection{Bayesian Analysis}

DFDA performs pattern recognition by estimating the probability density of the feature vector $\mathbf{X}$ for each class and then applying Bayes' rule. The data is assumed to consist of $n$ cases of $(C, \mathbf{X})$ pairs where $C \in(1,2, \ldots, J)$ is the class and $\mathbf{X}$ is a $p$-dimensional feature (predictor) vector. According to Bayes' rule, the probability that the class $C=C_{i}$ for a given value of the feature vector $\mathbf{X}$ and for prior class probabilities $p\left(C_{i}\right)=P(C=i)$ is (for the case that all features have discrete values)

$$
P\left(C=C_{i} \mid \mathbf{X}=\left(x_{1}, x_{2}, \ldots, x_{p}\right)\right)=A \cdot P\left(\mathbf{X} \mid C_{i}\right) \cdot p\left(C_{i}\right) .
$$

In the above equation, $\mathbf{X}$ is the feature vector to be classified, $P\left(\mathbf{X} \mid C_{i}\right)$ is the probability of observing $\mathbf{X}=\left(x_{1}, x_{2}, \ldots, x_{p}\right)$ when the true class is $C=C_{i}$, and $A=1 / P(\mathbf{X})$ is a constant that does not depend on the class. If some of the features are real-valued, the concept is identical, but the notation includes integrals. The predicted class for the feature vector will be the class that appears to maximize Eq. (1) on the basis of estimated $P\left(\mathbf{X} \mid C_{i}\right)$ and $p\left(C_{i}\right)$ (assuming equal misclassification costs).

Several well-known classifiers attempt to estimate $P\left(\mathbf{X} \mid C_{i}\right)$ by assuming some convenient distribution for the feature vector $\mathbf{X}$. For example, if we assume that $\mathbf{X}$ has a multivariate normal distribution with a different mean vector for each class, then Fisher's Linear Discriminant Analysis is the appropriate method. To estimate $P\left(\mathbf{X} \mid C_{i}\right)$, we simply estimate both the mean vector for each class and the covariance matrix (assumed to be the same for all classes). DFDA focuses on methods of estimating $P\left(\mathbf{X} \mid C_{i}\right)$ that make minimal assumptions about the distribution for the feature vector $\mathbf{X}$. We will include results from the application of a few parametric and other non-parametric methods for comparison to our non-parametric method.

\subsection{Density Estimation Using the Kernel Method}

We use the kernel method ${ }^{1}$ of density estimation. For each type of feature (continuous, categorical, and ordered categorical), we select a kernel density and show how to select a good value for the smoothing parameter in the kernel density. The density estimate for Class 1 of an input vector is the average of the product of the kernels evaluated only for the Class 1 cases in the training set. We repeat the calculation for each candidate class. 
To state things more formally, assume that we represent the training data for the Class 1 cases by

$$
D=\left\{\left(\mathbf{r}_{\mathbf{i}}, \mathbf{c}_{\mathbf{i}}, \mathbf{o}_{\mathbf{i}}\right) ; \mathbf{r}_{\mathbf{i}} \in R^{k}, \mathbf{c}_{\mathbf{i}} \in C^{l}, \mathbf{o}_{\mathbf{i}} \in O^{m} ; i=1, \ldots, n_{1}\right\}
$$

where $\mathbf{r}_{\mathbf{i}}, \mathbf{c}_{\mathbf{i}}$, and $\mathbf{o}_{\mathbf{i}}$ represent the continuous, unordered categorical, and ordered categorical components of feature vector $\mathrm{i}$ in data set $D$. The symbols $R^{k}, C^{l}$, and $O^{m}$ represent the spaces from which each of the components is drawn, so $k, l$, and $m$ are the number of features in the various components, and $n_{1}$ is the number of Class 1 examples in the training set. The equation for estimating the probability density function (p.d.f.) then becomes

$$
p\left(\mathbf{x}, \mathbf{y}, \mathbf{z} \mid D, \lambda_{r}, \lambda_{c}, \lambda_{o}\right)=\frac{1}{n_{1}} \sum_{i=1}^{n_{1}} K_{r}\left(x-X_{i}, \lambda_{r}\right) \cdot K_{c}\left(y-Y_{i}, \lambda_{c}\right) \cdot K_{o}\left(z-Z_{i}, \lambda_{o}\right)
$$

where $(x, y, z)$ is a typical vector in $R^{k}, C^{l}, O^{m} ; \lambda_{r}, \lambda_{c}$, and $\lambda_{o}$ are the smoothing parameters (possibly vector-valued) for the various density estimators; $\left(\mathbf{X}_{\mathbf{i}}, \mathbf{Y}_{\mathbf{i}}, \mathbf{Z}_{\mathbf{i}}\right)$ is the $i$ th feature vector of the $n_{1}$ Class 1 examples; and $K_{r}, K_{c}$, and $K_{o}$ are the kernels for the real, unordered categorical, and ordered categorical variables.

(Note: The kernel parameter list could also be given as $K_{r}\left(\mathbf{x}, \mathbf{X}_{\mathbf{i}}, \lambda_{r}\right)$ where we only show the variables used to calculate the kernel. However, we prefer the notation $K_{r}\left(\mathbf{x}-\mathbf{X}_{\mathbf{i}}, \lambda_{r}\right)$ because it clearly shows that we are computing some kind of difference between $\mathbf{x}$ and $\mathbf{X}_{\mathbf{i}}$.)

\subsection{Kernel Densities and Functions to Optimize the Smoothing Parameters}

In this section, we describe the functions $K_{r}, K_{c}$, and $K_{o}$ and show how to select good values for $\lambda_{r}, \lambda_{c}$, and $\lambda_{o}$. The implementation of the DFDA software has been broken up into phases; the equations shown have been implemented for Phase I. Phase I only provides one choice for each of the various functions. However, the software is written so that adding new functions later is straightforward.

\subsection{Kernel Functions}

\subsubsection{Continuous Features}

For $K_{r}$, we implemented the Multivariate Normal Density Function as shown in the following equation: 


$$
K_{r}\left(x^{\prime}\right)=(2 \pi)^{\frac{-k}{2}} \exp \left(-\frac{1}{2} x^{\prime} x^{\prime}\right)
$$

where $x^{\prime}$ equals $\left(\mathbf{x}-\mathbf{X}_{\mathbf{i}}\right) / \lambda_{r}$ and $x^{\prime T}$ is the transpose of $x^{\prime}$. We can also write Eq. (4) as

$$
K_{r}\left(\mathbf{x}-\mathbf{X}_{\mathbf{i}}, \lambda_{r}\right)=(2 \pi)^{\frac{-k}{2}} \cdot \mathrm{e}^{\frac{-\left(X_{i 1}-x_{1}\right)^{2}}{2 \lambda_{r}}} \cdot \ldots \cdot \mathrm{e}^{\frac{-\left(X_{i k}-x_{k}\right)^{2}}{2 \lambda_{r}}}
$$

where $x_{1}$ through $x_{k}$ are the values of the $k$ continuous features in $\mathrm{x}$, and $X_{i 1}$ through $X_{i k}$ are the values of the continuous features in $\mathbf{X}_{\mathbf{i}}$. Equation (5) is a specific choice for $K_{r}$ in Eq. (3).

In Phase $\mathrm{I}$, there is one smoothing parameter $\left(\lambda_{r}\right)$, one method of optimizing that smoothing parameter, and one kernel for all of the continuous features. In future phases, we will provide the functionality to have a different kernel and smoothing parameter for each feature.

\subsubsection{Unordered Categorical Features}

For $K_{c}$ we implemented the following relationship:

$$
K_{c}\left(c_{j} \mid x_{j}, \lambda_{c}\right)=\left\{\begin{array}{l}
\lambda_{c} \quad\left(c_{j}=x_{j}\right) \\
\frac{\left(1-\lambda_{c}\right)}{\left(n_{c_{j}}-1\right)}\left(c_{j} \neq x_{j}\right)
\end{array},\right.
$$

where $n_{c_{j}}$ is the number of categories for the $j$ th categorical unordered feature. Note that the larger the number of categories, the less weight that is given to the density estimation function when the feature values differ.

\subsubsection{Ordered Categorical Features}

The main properties we want for the kernel are that it be a p.d.f. and that it give less weight to unequal values that are farther away. Therefore, for $K_{o}$ we implemented the following:

$$
K_{o}\left(c_{j} \mid x_{j}, \lambda_{o}\right)=\left\{\begin{array}{l}
\lambda_{o} f\left(n_{c_{j}}\right) c_{j}=x_{j} \quad\left(c_{j}=x_{j}\right) \\
\frac{\left(1-\lambda_{o} f\left(n_{c_{j}}\right)\right) / d\left(c_{j}, x_{j}\right)}{\sum_{c_{j} \neq x_{j}}\left(1 / d\left(c_{j}, x_{j}\right)\right)}\left(c_{j} \neq x_{j}\right)
\end{array} .\right.
$$


Note that Eq. (7) reduces to Eq. (6) if $d\left(c_{j}, x_{j}\right)=1$ for all $c_{j} \neq x_{j}$, and $f\left(n_{c_{i}}\right)=1$.

\subsection{Methods to Optimize Smoothing Parameters}

In this section, we describe how to estimate the optimal smoothing parameters for each of the feature types. We ask the user to try a few "reasonable values" for the smoothing parameter. (We describe below how we determine what are "reasonable values" for the various smoothing parameters.) We display the misclassification rate for each choice. Phase I only uses one smoothing parameter for each type of feature, not for each individual feature. In order for one smoothing parameter to be sufficient, we have to standardize all the continuous features to have unit variance. We do this by dividing each continuous feature by its standard deviation to obtain unit variance. Future phases will use a different smoothing parameter for each feature.

\subsubsection{Continuous Features}

The user chooses the smoothing parameter so that it is between two extreme values obtained from Eq. (4.1.4) in Ref. 1 as follows:

$$
h_{\mathrm{opt}}=A(K) n^{-1 /(d+4)},
$$

where $A(K)$ is given by $\{4 /(2 d+1)\}^{1 /(d+4)}, n$ is the number of examples in the training set, and $d$ is the number of continuous features. We suspect that Eq. (8) with an actual value of $d$ will tend to produce $h$ values that are too large, so we will choose one extreme value of $h$ by putting $d=1$ (to make $h$ values smaller) in Eq. (8) and the other extreme value of $h$ by putting the actual value of $d$ in Eq. (8). These extreme values are displayed to the user before the user is prompted to enter his or her choice.

\subsubsection{Unordered Categorical Features}

Phase I will simply allow the user to input a value for $\lambda$, and we will enforce that the value be in the range $1 / 2 \leq \lambda \leq 1$. For future phases, we will allow a grid search for $\lambda$ in $1 / 2 \leq \lambda \leq 1$ with a user-specified value for the step parameter (e.g., increment $\lambda$ by 0.1 from 0.5 to 1.0 ).

\subsubsection{Ordered Categorical Features}

For Phase I, this will be handled in the same way as for the unordered categorical features. 


\subsection{Software Philosophy}

The fundamental principle underlying the Software Toolkit for Analysis Research (STAR) project ${ }^{2}$ at Los Alamos is that the technologies of extracting information from huge data sets can best be developed by cooperation between a team of specialists experienced with the general underlying computer science and mathematical problems and experts in the design and operation of specific operational systems. We are solving specific problems in such a way that the developed tools are as generally applicable as possible to new problem domains as they develop. This requires the development of a computational environment that ensures that all tools developed for tasks such as parsing, filtering, analyzing, and displaying can be easily applied to new situations. The use of STAR to implement the DFDA module is an example of how the STAR infrastructure can be used to facilitate both the development of an algorithm (e.g., kernel density estimation on mixed feature types) and the application of that algorithm to various data sets. We envision a uniform method of collection, storage, and analysis emerging as the benefits of utilizing the STAR software development philosophy become apparent.

The goal of STAR is to produce a research tool that facilitates the development and interchange of algorithms for locating phenomena of interest in large quantities of data. Using this toolkit, researchers can ascertain the most promising techniques, develop new and possibly more effective methods, and add or delete algorithms without major redesign work. The two primary software goals of the STAR system are that

1. it should be easy to add new algorithms to the system; and

2. it should be easy to apply algorithms already integrated into the STAR system to new applications (i.e., data sets).

We facilitate the first goal by providing generic data classes that allow the algorithm designer to focus on the algorithm development, not on the format and condition of the data. The algorithm developer only needs to know the public interface to the top-level data class, VectorData, to access the data in any way. The second goal is met by adopting easy-to-use file formats for the data and the attributes file. The attributes file allows the user to describe the structure of the data without requiring changes to software! (See Appendix C: File Formats on page 15.) For a new application, the user only needs to convert the data into the standard data file format (if it is not already in this format) and to provide an attributes file that describes the features in the data set. 


\subsection{Software Vision}

Our vision for software development considers what is best from both the developer's and the user's perspective. See Ref. 3 for an in-depth discussion of our vision for future software development.

\subsection{DFDA Results on Various Data Sets}

We compared the error rate obtained by DFDA on five data sets to the error rates obtained by several other classification techniques.

\subsection{Data Sets}

We briefly describe below the data sets used in our comparison. Each data set was broken into $75 \%$ training and $25 \%$ testing. See the table below for general information about each data set.

\begin{tabular}{|l|c|c|c|}
\hline \multicolumn{4}{|c|}{ TABLE 1. Data Sets Used in the Comparison } \\
\hline & $\begin{array}{c}\text { Number of } \\
\text { Cases }\end{array}$ & $\begin{array}{c}\text { Number of } \\
\text { Predictors }\end{array}$ & $\begin{array}{c}\text { Number of } \\
\text { Classes }\end{array}$ \\
\hline Digits & 1000 & 7 & 10 \\
\hline Attack & 1000 & 50 & 2 \\
\hline Waveform & 800 & 21 & 3 \\
\hline file5.df & 373 & 85 & 4 \\
\hline file6.df & 373 & 33 & 4 \\
\hline
\end{tabular}

\subsubsection{Digits}

The first data set mentioned in Table 1, Digits, has an unordered categorical class feature that can take values from 0 to 9 . These correspond to the digits that can be represented by a light-emitting-diode display on an ordinary hand calculator. There are seven binary feature values that indicate whether or not a segment of the display was on for that digit. A $10 \%$ error was randomly introduced into the feature values (i.e., a " 1 " was flipped to a " 0 " and vice-versa with a probability of .10). The theoretical optimal error rate on this data set is $26 \%$.

\subsubsection{Attack}

This is a simulated data set used to determine the effectiveness of algorithms at distinguishing between "normal" user behavior and "misuse" behavior on a computer system. The class feature is binary-valued with " 0 " indicating a normal audit trail record and " 1 " indicating a suspicious or misuse record. There are 50 binary features that can be used to discriminate between the two types of audit trail records. 


\subsubsection{Waveform}

The waveform data set consists of examples of three types of waveforms, and the best theoretical error rate that can be obtained is $16.0 \%$. The job of the classifier is to use features extracted from the waveform to correctly predict its type. There are 21 continuous features that can be used to distinguish among the various waveforms. In the following paragraph we describe in more detail how the waveform data set was generated.

Three triangular functions, $T_{1}, T_{2}$, and $T_{3}$, centered at $x=7, x=11$, and $x=15$, generate data for each of the three classes. To generate a Class 1 vector, we first generate a uniform random variable $u$ between 0 and 1 . Then $x_{m}=u \cdot T_{1}(m)+(1-u) \cdot T_{2}(m)+e_{m}$ where $e_{m}$, the error, is normally distributed with mean 0 and variance 1 , and $m=1,2, \ldots, 21$. To generate a Class 2 vector we use $T_{1}$ and $T_{3}$ as above, and to generate a Class 3 vector we use $T_{2}$ and $T_{3}$ as above.

\subsection{4 file5.df and file6.df}

These data sets contain readings obtained from particle detectors on satellites for detecting nuclear detonations. See Ref. 4 for more information about these data sets.

\subsection{Comparative Techniques}

We briefly describe the techniques to which we compared the DFDA method in this section. See Ref. 4 for details about these techniques.

\subsubsection{Classification and Regression Trees (CART)}

This is a commercial decision tree technique.

\subsubsection{Linear Discriminant Analysis (LDA)}

This is the original pattern recognition technique created by $R$. Fisher in the 1930s. LDA assumes that the data for each class has a multivariate normal distribution with the classes differing only in the mean vector.

\subsubsection{K-Nearest Neighbor Methods (K-NN)}

These techniques classify a given case in the testing set according to the classes assigned to the nearest (in the feature space) $k$ cases in the training set by using majority rule. In our experiments, we used the values of $1,3,5$, and 10 for $k$.

\subsubsection{Mixture Discriminant Analysis (MDA)}

This is a natural extension of LDA that allows any number of mean vectors, instead of just one, for a given class. 


\subsubsection{Flexible Discriminant Analysis (FDA)}

FDA attempts to exploit the fact that LDA can be derived by repeated linear regression of the class (viewed as a response) on the predictors. Then the linearity assumption is dropped, and any nonlinear regression methods (including neural networks) are allowed.

\subsubsection{Neural Network: Learning Vector Quantization (LVQ)}

The LVQ method finds a modest number of representative vectors (having the same dimension as each input vector) per class. The class of a given case is predicted by applying the K-NN method to these representative vectors rather than to the original data. During training, the representative vectors are adjusted to improve performance on the training data.

\subsection{Results}

Table 2 shows the error rates for all the techniques on the five data sets. DFDA was the best performer on the Digits data set, obtaining only a $24 \%$ error rate. The theoretical best on this data set is $26 \%$; we suspect that through "luck" the random fluctuations introduced on the data helped DFDA obtain a better than optimal result. The results for DFDA on file5.df and file6.df, although not the best, were at least comparable to most of the other techniques. DFDA was comparably worse than the other techniques for the Waveform and Attack data sets.

TABLE 2. Comparing Several Techniques to DFDA on Sample Data Sets

\begin{tabular}{|l|c|c|c|c|c|c|c|c|c|c|}
\hline Data Set & CART & LDA & 1-NN & 3-NN & 5-NN & 10-NN & MDA & FDA & LVQ & DFDA \\
\hline Digits & .28 & .25 & .88 & .87 & .85 & .89 & $.25^{\mathrm{a}}$ & .25 & .25 & .24 \\
\hline Attack & .10 & .18 & .35 & .31 & .28 & .25 & .18 & .18 & .19 & .31 \\
\hline Waveform & .31 & .18 & .27 & .20 & .20 & .16 & .19 & .16 & .18 & .25 \\
\hline file5.df & .17 & .16 & .18 & .17 & .17 & .19 & .21 & .07 & .10 & .15 \\
\hline file6.df & .15 & .17 & .18 & .17 & .14 & .17 & .18 & .12 & .14 & .19 \\
\hline
\end{tabular}

\subsection{Summary and Conclusions}

DFDA proved itself to be another viable method to try on a variety of data sets. DFDA, and density estimation in general, will often give superior results if sufficient training data is available. (The amount of training data needed before DFDA becomes optimal is an area for future work.) The software provides an environment in which kernel density estimation can be easily applied to data sets with both continuous and categorical features. The software also facilitates applying the DFDA algorithm to different data sets (simply create an attributes file for each new data 
set) and facilitates developing new algorithms based on the $\mathrm{C}++$ class infrastructure. Also, some of the methods benefit from a preliminary subset selection step (also called feature selection) that we have not conducted here.

\subsection{Future Work}

Using distribution-free density estimation on data that contain a mix of continuous, categorical, and ordered categorical features has not received extensive coverage in the statistical literature. Potential research topics include:

- How much training data is needed, as a function of the number of features, ranges, and domain sizes, for DFDA to outperform other techniques?

- Although there has been considerable work in determining effective density estimation techniques for distributions of continuous-valued features, there has not been much work on which of these techniques are effective when combined with other techniques that operate on categorical (unordered and ordered) features. Another area of future work is to determine the best categorical density estimators to use in combination with density estimators for continuous features.

- Given certain characteristics of the data set (e.g., covariance matrix, means, and variances), can we infer which kernels to use for the various feature types?

- Compare parametric to non-parametric approaches and study how much difference between actual and assumed distribution can be tolerated by parametric approaches before non-parametric approaches outperform them. Perform the comparison as a function of sample size and dimension of feature space.

- Define "effective dimension" of categorical features - in terms of when compared to sample size requirements for continuous features.

- Study advantages of transformations to uncorrelated features in terms of effective dimension reduction.

- Construct cross-validation methods for choosing the smoothing parameters that are tailored for pattern recognition. (We don't need to have the best possible estimate of each class density unless two or more class probability densities are nearly equal, so we don't need a global measure of closeness of estimated density to true density.)

- Compare joint search for smoothing parameter vector to one-at-a-time search.

- Experiment with subset selection methods as a preliminary step before applying DFDA. 


\subsection{Acknowledgments}

This report describes work performed during FY96 for the Knowledge Fusion project and was sponsored by the Department of Energy, Office of Nonproliferation and National Security (DOE NN-20). The STAR project mentioned in Section 5.0 on page 6 is funded by the NSA and DOE NN-20.

\subsection{References}

1. B. W. Silverman, Density Estimation for Statistics and Data Analysis, 1st Ed. (Chapman and Hall Ltd., London and New York, 1986).

2. T. L. Burr, J. E. Doak, P. Helman, B. Hoffbauer, J. A. Howell, P. M. Kelly, L. McGavran, R. Pitts, J. M. Prommel, T. R. Thomas, R. B. Strittmatter, and R. Whiteson, "Software Toolkit for Analysis Research (STAR)," Los Alamos National Laboratory report LA-12617-MS (1993).

3. Justin Doak, "Case Study on Selecting an Environment for Software Development," Los Alamos National Laboratory, Safeguards Systems Group report LA-13308-MS (May 1997).

4. Tom Burr, Justin Doak, Jo Ann Howell, Dave Martinez, and Richard Strittmatter, "Knowledge Fusion: Time Series Modeling Followed by Pattern Recognition Applied to Unusual Sections of Background Data," Los Alamos National Laboratory report LA-13075-MS (March 1996).

\subsection{Appendix A: Obtaining, Installing, and Using the Software}

To use the software, you must have a $\mathrm{C}++$ compiler with true template support (not just a cfront interpreter) and Rogue Wave's tools.h+t (see http://www.roguewave.com). To obtain the software, go to http://www-safeguards.lanl.gov' infoanal.html and download the compressed tar file (DFDA.tar.Z) that contains a tutorial, "dfda_tutorial.txt," the C++ source, sample data and attributes files, and a makefile. If you have any questions about software installation and use, contact Justin Doak (justin@lanl.gov).

\subsection{Appendix B: DFDA Software}

We assume that data is passed between modules using ascii files and that the format of these files is described in an attributes file. Data classes have been written that read in an attributes file, use the information from the attributes file to read in the 
data files, and then calculate various statistics on features in the data set. The attributes file contains such information as the types of features, their names, the ranges of continuous features, and the domains of categorical features. With this necessary information, we can parse the data files and read them into $\mathrm{C}++$ classes. These $\mathrm{C}++$ classes provide functions to compute statistics on the data and generate new features (e.g., calculate the standard deviation for a feature, then divide all values by this to standardize the feature to unit variance). This is a large portion of the Information Preparation functionality of STAR. Note that NO rewriting of code is necessary with new data sets; one only needs to write an attributes file for each new data set to be analyzed.

We have approached the software implementation in phases. For each phase shown below, we list the functionality (often with some indication of future functionality), assumptions, user interface, output, and error handling implemented in that phase. Phase I has been completed.

\subsection{Phase I}

- We assume that the data is already split into a training and a testing set. (Twothirds training and one-third testing is a typical split.) Future phases will allow us to enter one data set and do various types of sampling to create the training and testing sets.

- Phase I provides only one choice for the density estimation techniques and for selecting values for the smoothing parameters. Future phases will add choices for these parameters.

- Output, initially, is just the error rate. Eventually, we will output other things like the confusion matrix, a list of misclassified indices, the misclassification cost, and a log of options chosen.

- An attributes file is required that describes the features in the data set. Some of the attributes for each feature may be the type of feature, the range of continuous features, and the possible values for categorical features. See "Format of Attributes File" on page 15 for a more complete description.

- Phase I assumes that there are no missing values in the data set. We assume that rationalization (i.e., correction of missing and erroneous values) occurs in a separate operator before this one. (We may do some rationalization within this module in Phases II or III, if we do not have a separate rationalization operator at that time.)

- We assume that all continuous features have been standardized to have unit variance. We calculate the standard deviation for each feature and divide all feature values by this to obtain unit variance. For future phases, we may want to try things without standardizing to unit variance, so we will need to keep the original values. 


\subsubsection{User Interface and Output}

The following is a list of what the user sees.

- Choices of smoothing parameter for the categorical, continuous, and ordered categorical density estimators. The smoothing parameters for the categorical and ordered categorical kernels must be between 0.5 and 1.0. A range of acceptable values is presented to the user for the smoothing parameter in the continuous kernel. The user then selects a value within this range.

Future phases may be able to calculate smoothing parameters that depend on each other (i.e., the calculation of the smoothing parameter for the continuous features depends on the smoothing parameter for the categorical features). Note that for Phase I the user will only be able to set a single smoothing parameter for each type of feature. Eventually, we will add the functionality for calculating smoothing parameters separately for each feature. (This implies that the density will be calculated separately for each feature, as it currently is.)

- Choice of density estimator for each of the various types of data. Phase I only implements one choice per feature type.

- Enter a training set filename and a testing set filename. In future implementations, the user may choose to enter the name of one large filename; the system will perform some type of sampling to create the training and test sets according to the user's specifications. We should at least provide the ability to break the training and testing set into user specified sizes (e.g., 50-50) or do leave-one-out cross validation (useful for small data sets).

- Enter the name of the attributes file. The user only needs to enter a base filename. The system assumes that files of *.trn, *.tst, and *.attr are available.

- Enter the prior probabilities for the various classes. (1) The user assumes the prior probabilities are all equal; (2) the user manually sets the prior probabilities (must sum to 1.0); and (3) the system calculates the prior probabilities from the training set.

Phase I only outputs the error rate. Future phases may output, for example, the misclassification cost, the confusion matrix, the indices of misclassified examples, and the predicted classes for all vectors in the testing set.

\subsubsection{Error Handling}

This is not a high priority for the DFDA module. However, we would eventually like to incorporate certain error handling functionality into this module.

- The smoothing parameter for either categorical or ordered categorical is not between 0.5 and 1.0. This is completed in Phase I.

- Filename doesn't exist. If the training, testing, or attributes files are not present, we should print out or display the appropriate error. This is completed in Phase I. 
- Format or syntax error in one of the input files. This is not implemented in Phase I.

- Prior probabilities do not sum to 1.0. If the user enters the prior probabilities, we add them up to make sure they total to 1 . This is implemented in Phase I.

\subsection{Phases II and III}

This is our wish list for functionality to add to the system.

- The user will be able to select costs for the various types of error. For example, in the two-class problem, guessing Class 1 when the actual Class is 2 may be considered more serious than guessing Class 2 when the actual Class is 1 .

- Devise a method to calculate standard deviation for ordered and unordered categorical features.

- Add the ability to calculate the range for continuous features and the domain of unordered categorical features from the training set. (In the case of unordered categorical features, we are trying to determine all the values in the domain to enable future error checking and to get an estimate of the number of possible values for the category.) Below, we list options for calculating the range of continuous features from the training set.

- Calculate the trimmed mean and the trimmed standard deviation using the middle $90 \%$, for example, of the data and flag cases that are beyond four times the trimmed standard deviation. This would just be a flag because we have not determined if we will treat those cases as having erroneous data (bogus data). We may just notify the user that there are suspicious values.

- Calculate the range in the training data and use that range plus $10 \%$ (selectable parameter) for future theoretical ranges in cases for which we get only part of the data at a time.

- Do bounds checking for bogus values and correct them. We will also fill in missing values in some reasonable fashion.

- Log, in an output file, which options were chosen.

- Add more options for ordered categorical feature types.

(1) Ordered Categorical. Distance is determined by the difference in position between the two values in the ordering. For example, with possible values of $1,5,17$, and 91 , the distance between 1 and 17 is 2 because their positions are 1 and 3 . This is probably the most common way of handling ordered categorical features and is implemented in Phase I.

(2) Numeric Ordered Categorical. Distance is determined by subtracting the actual values. For example, with possible values of $1,5,17$, and 91 , the distance between 1 and 17 is 16 . 
(3) User-Specified Distance Ordered Categorical. The user specifies the distance between the values. The sum of these distances needs to be calculated to determine the distance between any two values. For example, with possible values of 1(3) 5 (1) 17 and (2) 91, the distance between 1 and 17 is 4 . The quantity $(x)$ denotes the distance between two feature values.

(4) Distance Matrix Categorical Feature. The distance between any two values is determined by looking up the value in a table. For example, with possible values of $1,5,17$, and 91 on the table shown below, the distance between 1 and 17 is 15 .

\begin{tabular}{|r|r|r|r|r|}
\hline \multicolumn{5}{|c|}{ Sample Matrix for a Categorical } \\
\hline & 1 & 5 & 17 & 91 \\
\hline 1 & 0 & 3 & 15 & 6 \\
\hline 5 & 3 & 0 & 51 & 32 \\
\hline 17 & 15 & 51 & 0 & 2 \\
\hline 91 & 6 & 32 & 2 & 0 \\
\hline
\end{tabular}

Note that we will use strings to represent the types given by (1), (3), and (4) and reals for (2).

\subsection{Appendix C: File Formats}

\subsection{Format of Data Files}

The data format, for all phases, is shown below.

class_value feature_value_l $1 .$. NA ... feature_value_n $n$

The value for the class variable is always the first value on a line; values are space delimited; missing feature values are denoted by "NA"; and there is one vector of values per line (i.e., a single vector is not split over multiple lines). Note that the Phase I implementation does not deal with missing data.

\subsection{Format of Attributes File}

Phase I will allow the user to have two kinds of attributes files. The first kind of attributes file requires the user to enter a line corresponding to every feature, including the class feature, in the data set. The syntax for a file of this variety follows. (The comments are for clarification only and would not appear in an actual attributes file.) 
// Class attribute goes here, whichfor now must be // unordered categorical.

cat name_of_class_feature value_l $1 .$. value_n

// A continuous feature with range explicitly specified. cont feature_name begin_range end_range

// A continuous feature without a range specified. cont feature name

// An unordered categorical feature. cat feature_name value $\_1 .$. value $\_n$

// An ordered categorical feature. ord_cat feature_name value_1 . . value_n.

Here is a sample attributes file of Type I.

cat ball_type basketball baseball tennisball

cont diameter 0.012 .0

cont weight

cat texture seamed fuzzy dimpled

ord_cat lifespan day week year

Note that we have a class feature that is of the type unordered categorical, two continuous features, one unordered categorical feature, and one ordered categorical feature. The second continuous feature, weight, does not specify a range; the system is able to read in the actual (theoretical) range if given and will set default values otherwise.

The second type of attributes file assumes that all the features are continuous except for those explicitly specified as categorical. (Continuous variables may also be explicitly specified if the user wishes to assign a name or an actual range to a continuous feature.) Here is the syntax for this type of attributes file.

number_of_features

feature_number cat feature_name feature_value_1 . . f feature_value_ $n$

feature_number ord_cat feature_name feature_value_1 . . f feature_value_n

feature_number cont feature_name [begin_range end_range]

// more attributes can go here. 
Here is an example of this type of attributes file:

7

0 cat ball_type basketball baseball tennisball

1 cont weight 100.0250 .0

4 ord_cat lifespan day week year

We have seven features; the first is an unordered categorical feature that represents the class feature, the fifth is an ordered categorical variable, and the rest are continuous variables. Feature 1 , weight, is continuous and has been explicitly specified so that it could be named and assigned a range. The main advantage to this version of the attributes file is that, for data sets with many continuous features, less work needs to go into the attributes file.

Type I and II attributes files are distinguishable by the fact that Type I will begin either with the keywords "cont," "cat," or "ord_cat," but Type II will begin with an integer. The system automatically distinguishes between these two types of files.

Notes. For continuous features (at least for density estimation), there is no need to require the user to enter the ranges other than to do some variety of error checking on values. The user will, on the other hand, need to enter the domain for ordered categorical features because the system must know the ordering to calculate the difference. Note that it is only necessary to get the number of categories from the user for the unordered categorical features. However, at least for Phase I, we will require that the user enter all the values for a categorical feature in the attributes file primarily so we can use the values later to do error checking. We may try to pull the domain for unordered categorical features from the training set in a future phase. 